\title{
Philosophiques
}

\section{Topologie et modélisation chez René Thom : l'exemple d'un conflit de valeurs en éthique}

\section{Yanick Farmer}

Volume 37, numéro 2, automne 2010

URI : https://id.erudit.org/iderudit/045188ar

DOI : https://doi.org/10.7202/045188ar

Aller au sommaire du numéro

Éditeur(s)

Société de philosophie du Québec

ISSN

0316-2923 (imprimé)

1492-1391 (numérique)

Découvrir la revue

Citer cet article

Farmer, Y. (2010). Topologie et modélisation chez René Thom : l'exemple d'un conflit de valeurs en éthique. Philosophiques, 37(2), 369-386.

https://doi.org/10.7202/045188ar
Résumé de l'article

Pour l'épistémologue, mais aussi pour le théoricien de l'éthique, la théorie thomienne des modèles présente un intérêt particulier. Elle établit que la connaissance, ainsi que toutes les catégorisations abstraites qui en résultent, sont façonnées en première instance par les morphologies d'événements qui s'offrent à nos sens. L'intérêt de cette approche épistémologique pour l'éthique est de répondre au problème de son universalisation. Alors que l'éthique traditionnelle fonde la justification sur des principes qui, linguistiquement ancrés, peinent à s'imposer au-delà des cultures particulières, la théorie thomienne des modèles ouvre la voie à un nouveau type d'aide à la décision qui est fondé sur un mode de connaissance universel : la topologie. Cet article expose les fondements épistémologiques de la théorie thomienne, pour ensuite en proposer une application originale aux conflits éthiques. 


\title{
Topologie et modélisation chez René Thom: l'exemple d'un conflit de valeurs en éthique
}

\author{
YANICK FARMER \\ Groupe de recherche en bioéthique \\ Université de Montréal \\ yanfarmer@yahoo.ca
}

\begin{abstract}
RÉSUMÉ. - Pour l'épistémologue, mais aussi pour le théoricien de l'éthique, la théorie thomienne des modèles présente un intérêt particulier. Elle établit que la connaissance, ainsi que toutes les catégorisations abstraites qui en résultent, sont façonnées en première instance par les morphologies d'événements qui s'offrent à nos sens. L'intérêt de cette approche épistémologique pour l'éthique est de répondre au problème de son universalisation. Alors que l'éthique traditionnelle fonde la justification sur des principes qui, linguistiquement ancrés, peinent à s'imposer au-delà des cultures particulières, la théorie thomienne des modèles ouvre la voie à un nouveau type d'aide à la décision qui est fondé sur un mode de connaissance universel: la topologie. Cet article expose les fondements épistémologiques de la théorie thomienne, pour ensuite en proposer une application originale aux conflits éthiques.
\end{abstract}

\begin{abstract}
For both the epistemologist and the ethicist, René Thom's model theory is of particular interest. It assumes that knowledge, as well as all forms of abstract categorizations, are shaped by the morphologies that we perceive. The benefit of Thom's epistemological approach is then to contribute to the problem of universalization in ethics. Whereas traditional theories in ethics elaborate justification with language-based principles that cannot be applied universally, René Thom's model theory leads to a new modeling of ethics based on a universal form of knowledge: topology. This paper sets forth the epistemological foundations of Thom's theory, and then proposes an application to ethical conflicts.
\end{abstract}

\section{Introduction}

À ceux, dans les collèges et les universités, qui souhaitent s'initier à la philosophie et connaître quelle est la tâche ultime du philosophe, on a coutume de présenter cette image forte exprimée par Platon dans la République ${ }^{1}$ : l'allégorie de la caverne. Le philosophe grec y représente les hommes au fond d'une caverne, enchainés par les jambes et par le cou, et donc incapables de se lever ou de se retourner. Tout ce qu'ils peuvent voir, depuis leur enfance, est devant eux. Ce sont les ombres des choses du monde qui se projettent sur le fond de la caverne grâce à la lumière d'un feu situé à l'entrée de celle-ci. La réalité de ces hommes, nous dit Platon, est en fait la nôtre. Par nature et par les limites que nous imposent les sens, nous sommes pour ainsi dire condamnés à ne voir, directement, que la surface des choses.

1. Voir Platon, La République (trad. Robert Baccou), Paris, Flammarion, 1966, p. 273-276. 
Pour Platon, c'est précisément au cœur de cette situation que s'amorce la tâche ultime du philosophe: celle de pousser les hommes, par le questionnement et la connaissance, à s'élever au-delà des apparences pour embrasser le réel, c'est-à-dire le vrai et par conséquent le bien. Mais comment y arriver? Comment des hommes enchaînés et immobilisés au fond d'une caverne peuvent-ils, uniquement à travers ce regard qui les porte sur des ombres, approcher le vrai et le bien? À cette question difficile, mais essentielle, qui souligne la difficulté d'une science qui s'élève sur l'expérience immédiate des objets qui nous entourent, le mathématicien et philosophe français René Thom apporte une réponse à la fois originale et philosophiquement solide: la théorie des modèles, mieux connue sous le nom de théorie des catastrophes ${ }^{2}$. À travers Thom, comme nous le verrons, ce n'est pas seulement le vieil idéal platonicien d'un ordre mathématique transcendant et premier qui revit et s'actualise, mais aussi celui d'une philosophie qui, renonçant au cloisonnement de la pensée par la langue naturelle, s'ouvre à la profondeur sémantique favorisée par l'adjonction de nouvelles formes de modélisation inspirées des mathématiques.

Pour l'épistémologue, mais aussi pour le théoricien de l'éthique, la théorie thomienne des modèles, comme je l'appellerai dans la suite de ma réflexion, présente un intérêt tout particulier. Elle établit que la connaissance, ainsi que toutes les catégorisations abstraites qui en résultent, sont façonnées en première instance par les morphologies d'événements qui s'offrent à nos sens, et que les mots et les concepts de la langue naturelle se constituent, pour ainsi dire, à la surface de ces morphologies. À mon sens, et cette idée sera la trame de cet article, l'intérêt de cette approche épistémologique pour l'éthique est de répondre au problème de son universalisation. Alors que l'éthique traditionnelle, en santé publique par exemple, fonde la prise de décision sur des principes qui, linguistiquement ancrés, peinent à s'imposer au-delà des cultures particulières ${ }^{3}$, la théorie thomienne des modèles, c'est du moins la thèse que je veux défendre, ouvre la voie à un

2. Il convient ici de rappeler que dans l'ouvrage initial où il expose sa théorie, Thom parle d'une théorie générale des modèles, et non d'une théorie des catastrophes. C'est à la suite des divers développements qu'a connus la théorie, notamment sous l'impulsion du mathématicien anglais Erik Christopher Zeeman, que le terme "théorie des catastrophes" (TC) s'est imposé. Étant donné que le terme modèle est d'usage courant, contrairement au mot catastrophe, et que la portée de la théorie de Thom est très générale, j'ai choisi, dans cet article, d'utiliser plutôt l'expression "théorie des modèles". Voir René Thom, Stabilité structurelle et morphogenèse. Essai d'une théorie générale des modèles (2 éd.), Paris, InterEditions, 1977, page titre.

3. Par exemple, la notion d'autonomie, qui est centrale en bioéthique et en santé publique, n'a pas la même signification dans toutes les cultures. Voir à ce sujet l'intéressante étude menée par la Direction de la santé publique de Montréal: Marie-Ève Bouthillier, «Autonomie et responsabilité: qui décide en matière de santé ?", dans Alex Battaglini (dir.), Les services sociaux et de santé en contexte pluriethnique, Montréal, Direction de la santé publique, 2010. 
nouveau type d'aide à la décision qui est fondé sur un mode de connaissance universel: la topologie. L'idée-force que je souhaite mettre de l'avant est celle d'une géométrisation des problèmes éthiques. Par ce moyen, je veux suggérer que plus la justification éthique est localisée (spatialisée), plus il est possible de l'universaliser. À l'inverse, plus on s'éloigne d'une théorie locale, plus on expose le discours éthique au risque d'un relativisme induit par la sémantique culturellement située des langues naturelles.

Dans la suite de cette réflexion, je vais d'abord procéder à l'exposé des fondements épistémologiques de la théorie thomienne, pour ensuite en proposer une application originale aux conflits éthiques. Je veux ainsi faire valoir, dans un premier temps, la contribution plus large de Thom à l'intelligibilité des conflits de valeurs ou de principes en sciences humaines et sociales. Je souhaite également, dans un deuxième temps, faire ressortir les avancées philosophiques permises par la théorie thomienne des modèles, ainsi que les lignes de l'immense programme philosophique qu'elle ouvre, dans certains de ses prolongements.

\section{Le passage de l'apparent au réel: les fondements de l'approche épistémologique thomienne}

Avant d'entrer dans l'examen de la méthode elle-même, je veux faire remarquer, puisque j'avancerai dans cette partie de mon exposé à l'aide d'une analogie entre les pensées de Platon et de Thom, que dans l'allégorie de la caverne, les choses du monde n'apparaissent au regard des prisonniers qu'à la condition d'être éclairées par la lumière, celle du feu qui brûle derrière eux. Chez Thom, il me semble que ce feu correspond à l' "étincelle ", c'està-dire à l'intérêt et à la passion qui sont au fondement de tout effort sincère vers la connaissance. Cette étincelle fait apparaître un phénomène - une ou des ombres - comme un problème qu'il faut expliquer. C'est, je dirais, la première étape, la voie d'accès à la théorie des modèles énoncée par Thom.

Dès lors, une deuxième condition surgit pour faire advenir ce problème à la pensée. C'est que pour reconnaître un problème, un phénomène à expliquer, il faut le distinguer et le détacher d'un espace ambiant. Mais comment cela se fait-il ? Cela est possible parce que les objets, les choses ou les phénomènes en général possèdent un bord. Pour Thom toute perception, et donc toute théorisation, débute par une discontinuité phénoménologique, par une singularité qui apparaît dans l'espace-temps de la perception. Ce point où se produit une rupture de l'ordre topologique ambiant est à l'origine de la catastrophe, qui est un changement soudain de l'ordre d'un phénomène ${ }^{4}$.

L'espace où apparaissent les phénomènes - les ombres de Platon s'appelle, dans la théorie des modèles de Thom, l'espace substrat. Dans

4. Pour une introduction vulgarisée à la notion de catastrophe, voir René Thom, Prédire n'est pas expliquer, Paris, Flammarion (coll. "Champs»), 1993, p. 28-29. 
l'allégorie de la caverne, l'espace substrat correspond à la surface du mur sur laquelle les ombres sont projetées. Dans notre vie quotidienne, cet espace est l'espace euclidien à trois dimensions auquel on ajoute le temps.

Cependant, pour s'assurer de bien comprendre un phénomène problématique, il faut d'abord l'isoler et le définir. On peut y arriver en plaçant mentalement le phénomène problématique dans une espèce de "boîte". À l'intérieur de cette boîte, il est alors possible pour l'observateur de manipuler le phénomène à sa guise. Ainsi, en observant l'évolution du phénomène isolé, l'observateur sera mieux à même d'en saisir la nature. Dans le processus de modélisation élaboré par Thom, il faut, à cette étape, créer un espace de paramètres, appelé aussi espace de contrôle. Il s'agit donc pour constituer cet espace de trouver des paramètres dont la variation permettra d'établir un modèle du phénomène, c'est-à-dire d'en tirer une structure géométrique continue, descriptible en langue naturelle, et donnant à construire des hypothèses, des théorèmes ou peut-être même des lois.

En faisant varier les paramètres de contrôle et en notant les comportements résultant de cette action, l'observateur établit en fait une topologie du phénomène observé qu'il peut situer à l'aide d'un système de coordonnées $(a, b, x)$, où et « $a$ » et « $b$ » sont des paramètres de contrôle et « $\mathrm{x}$ » le comportement observable du phénomène. À partir de là, il est donc possible de définir une fonction algébrique et une topologie (ou un graphe) qui la représente. Certaines variables du phénomène observé sont dites «internes » et sont inaccessibles à l'observateur. Les variables «externes» sont les paramètres contrôlés par celui-ci ${ }^{5}$. Suivant cette approche, l'objectif de la modélisation est de partir d'une situation locale — le phénomène - et de déduire la dynamique globale qui lui donne naissance: «La théorie des catastrophes suppose justement que les choses que nous voyons sont seulement des reflets et que pour arriver à l'être lui-même il faut multiplier l'espace substrat par un espace auxiliaire et définir dans cet espace produit l'être le plus simple qui donne par projection son origine à la morphologie observée ${ }^{6}$.»

Il est alors aisé de revenir à l'analogie avec l'allégorie de la caverne. La théorie thomienne des modèles, et l'approche épistémologique qui la soustend, répondent au problème des prisonniers de la caverne. Le découpage par le regard d'une morphologie spécifique des phénomènes apparents renvoie, par projection sur un espace de paramètres défini par l'observateur, à un être géométrique qui existe a priori, et qui constitue au fond, comme nous le verrons, la condition même de l'apparaître des phénomènes et de

5. Cette approche dite «herméneutique ", qui part des aspects les plus saillants de l'apparaître phénoménologique pour remonter vers un modèle qui engendre cette morphologie, est appelée deuxième voie de la TC. Sur cette distinction, voir Jean Petitot, «Présentation», dans Jean Petitot (dir.), Logos et théorie des catastrophes, Genève, Éditions Patino (coll. "Colloque de Cerisy»), 1988, p. 46-47.

6. René Thom, Paraboles et catastrophes, Paris, Flammarion (coll. "Champs»), 1983, p. 85 . 
leur descriptibilité en langage ordinaire. En outre, la méthode proposée par Thom prend acte de la position épistémique particulière dans laquelle se retrouve le prisonnier enchaîné, de même que du statut ontologique des phénomènes soumis à des niveaux multiples de description ${ }^{7}$. Elle se refuse à une approche réductionniste par laquelle l'observateur doit se saisir des phénomènes et les «casser» en entités élémentaires dont la combinatoire est censée en révéler toute la complexité. Au lieu de quitter l'espace où apparaissent les phénomènes - l'espace substrat — pour chercher vainement à maîtriser la multitude des paramètres internes qui en contrôlent l'évolution (la position, la vitesse, la température, etc.), l'approche thomienne choisit d'ignorer cette variabilité interne qui se dérobe au regard et de se limiter à l'examen d'un nombre restreint de paramètres externes jugés essentiels ${ }^{8}$. Ce procédé aboutit ensuite à la détermination d'une structure topologique obtenue par le prolongement du phénomène sur l'espace auxiliaire des paramètres externes.

Je propose maintenant de partir de cette analogie et de ces principes brièvement esquissés et d'appliquer la théorie thomienne des modèles à la compréhension d'un conflit éthique. Cette démarche me conduira, par la suite, à la mise en évidence d'avancées philosophiques plus générales permises par la théorie.

\section{Une application de la théorie thomienne des modèles à un conflit éthique: le cas de l'opposition entre privatisation et étatisation du système de santé}

\subsection{Fondements du modèle}

Dans l'application de la théorie que je propose dans la suite de mon exposé, je tenterai de rattacher les tensions et les discontinuités qui se font jour à l'occasion d'un conflit ou d'un dilemme éthique à une figure topologique qui en détermine les contraintes et la morphologie. L'objectif poursuivi par la modélisation des conflits est, pour reprendre les mots de Thom, de «réduire l'arbitraire de la description » et de contribuer à la mise en place d'une typologie des conflits qui, à son tour, pourrait favoriser la création d'algorithmes de décision.

Je choisirai pour l'application de la théorie thomienne des modèles l'exemple d'un conflit de valeurs extrêmement actuel et connu de tous dans notre société, à savoir l'opposition entre la privatisation et l'étatisation du

7. Contrairement à l'approche réductionniste qui opère toujours à l'intérieur d'un même espace de description des phénomènes, en renvoyant simplement l'objet complexe à la description d'interactions entre composants «simples» et "visibles» (les molécules, les particules, les processus physico-chimiques, etc.), la théorie thomienne des modèles crée des niveaux de description variables grâce aux espaces multipliés permis par une modélisation topologique des phénomènes.

8. À l'échelle macroscopique des catastrophes élémentaires, ces paramètres sont au nombre de quatre ou moins. 
système de santé. Ainsi, suivant les étapes du processus de modélisation esquissé précédemment, il me faut d'abord identifier avec précision le phénomène que je veux modéliser. Pour le cas que je souhaite examiner, il me semble approprié de prendre l'action gouvernementale en matière de financement (public ou privé) des soins de santé comme phénomène apparent dans lequel s'illustre un dilemme éthique. Ce choix se justifie par le fait que les actions et les comportements des agents moraux sont les aspects proéminents de la morphologie des conflits, et qu'ils se prêtent alors plus naturellement à une modélisation topologique.

La tension qui se manifeste entre la privatisation et l'étatisation représente ce que la théorie des modèles de Thom appelle un conflit d'attracteurs ${ }^{9}$. Puisque la notion d'attracteur est avant tout mathématique, je la traduirai par une autre expression, plus commune, et sans doute aussi plus conforme au type de modélisation que je propose. Je dirai que ce conflit d'attracteurs est un conflit de tendances ${ }^{10}$. Mathématiquement, ces tendances sont des potentiels minimaux qui en quelque sorte "attirent» le système dans leur direction et déterminent son comportement visible. Le minimum de potentiel est la caractéristique des phénomènes structurellement stables qui, de ce fait, sont descriptibles linguistiquement. Étant donné que la modélisation entreprise ici débute par une observation de phénomènes stables et reconnaissables, il est alors compréhensible qu'elle soit associée à une mathématique de potentiels.

Dans le cas d'un conflit éthique, les valeurs sont souvent les attracteurs du système de relations qui s'établit entre deux agents moraux. La situation peut être plus complexe, mais dans l'exemple qui nous occupe, deux attracteurs orientent l'action gouvernementale. Il y a premièrement l'étatisation, comprise comme transfert sous l'autorité de l'État de la gestion du système de santé; puis il y a la privatisation, entendue comme transfert vers l'entreprise privée de la gestion de ce système. Mais à un instant donné de l'évolution du système, un seul de ces deux attracteurs est visible, au sens où l'action gouvernementale sera catégorisée par son appartenance, à des degrés d'intensité variables, à l'une ou l'autre de ces deux tendances.

En face d'un tel conflit, plusieurs questions peuvent émerger quant aux comportements des acteurs du conflit: qu'est-ce qui détermine le choix du gouvernement d'aller vers la privatisation ou l'étatisation du système de santé? Pourquoi l'action gouvernementale sur ces questions peut-elle évoluer continûment ou parfois brutalement? L'opinion publique est-elle

9. Dans l'étude des systèmes dynamiques, un attracteur, appelé aussi ensemble-limite, est un ensemble, une courbe ou un espace vers lequel un système tend de façon irréversible. Si on prend l'exemple d'un pendule qui oscille en l'air, l'attracteur du système formé par le pendule sera un point, car ce sera la position finale du pendule qui subit le frottement de l'air. La détermination des attracteurs d'un système permet donc d'en définir le comportement.

10. L'usage de ce mot est d'ailleurs accepté par Thom lui-même. Voir Thom, Prédire n'est pas expliquer, p. 44. 
l'unique base de décision? Existe-t-il des contraintes structurelles qui ne sont pas nécessairement économiques ou politiques?

\subsection{La réalisation du modèle}

Du point de vue de la théorie des modèles de Thom, ces questions font intervenir une espèce de «dynamique des variations ${ }^{11}$. C'est pourquoi les questions posées plus haut peuvent se résumer à la question suivante: $\mathrm{y}$ a-t-il une structure générale stable à partir de laquelle il est possible de produire des hypothèses et des énoncés qui expliquent la forme de ces variations? Comme je l'ai expliqué dans la seconde partie de mon exposé, il faut dans un premier temps, pour répondre à cette question, définir un espace de paramètres (espace de contrôle) à partir duquel il deviendra possible de contrôler et d'observer les variations de comportement à l'intérieur du système, c'està-dire, pour le cas qui nous concerne, l'action gouvernementale en matière de financement des soins de santé. Il faut donc trouver des paramètres dont la variation influence l'action gouvernementale dans le sens de l'un des attracteurs.

Je propose que le premier de ces paramètres soit l'égalité. En effet, il semble que ce soit principalement au nom de l'égalité que certains citoyens ou élus réclament le maintien du système de santé public et universel. À l'autre extrémité, on retrouve plutôt un système de santé qui, pris en charge par l'entreprise privée, valorise plutôt la liberté individuelle, donc la possibilité pour un usager du système de payer pour avoir accès, quand il le veut, à des soins adaptés à ses besoins propres. Ainsi, plus la notion d'égalité prend de l'importance au sein d'une société, plus le gouvernement aurait tendance, poussé par la force de l'opinion publique, à privilégier un système de santé public et universel (étatisé).

Il paraît assez clair que la variation de l'importance que prend l'égalité pour une société permet d'expliquer la force de l'attracteur "étatisation" dans ce conflit éthique. Mais est-ce suffisant? Certainement pas. Il suffit pour s'en convaincre d'observer des sociétés où la tendance vers la privatisation du système conserve un pouvoir d'attraction très puissant, même si l'égalité y est promue et protégée. Il devient donc tout aussi clair que la variation unidimensionnelle de l'importance accordée à l'égalité ne peut tout expliquer. Mais alors quel paramètre peut expliquer, par exemple, l'émergence parallèle d'un système de santé privé dans un contexte social et politique où domine la valeur d'égalité?

Je crois que ce paramètre, ce sont les coûts du système. Cette hypothèse me semble justifiée par le fait que si les coûts du système "explosent ", autrement dit si l'État n'a plus les moyens de se payer un système de santé

11. J'ai choisi cette expression pour traduire en langage courant l'emploi, chez Thom, du concept de «dynamique de gradients». En mathématiques, le gradient est une quantité représentant la variation d'une fonction par rapport à la variation de ses paramètres. 
universel (égalitaire), il est probable que cet État choisisse de limiter le financement public des soins de santé, malgré l'importance que la société donne à l'égalité. Le paramètre des coûts aide alors à comprendre pourquoi la variation de l'action gouvernementale le long de l'axe égalité n'est pas toujours continue. Comme nous allons le voir, le paramètre des coûts ajoute une autre dimension à la modélisation de ce conflit éthique.

Je dirais qu'en première approximation, avec l'utilisation de ces deux paramètres, il devient possible de constituer la «boîte » de l'espace de contrôle et de reproduire le conflit éthique et ses variations. Or, dans la théorie des catastrophes dites "élémentaires " ${ }^{12}$, un conflit ayant un paramètre interne noté « $\mathrm{X}$ » (action gouvernementale), et deux paramètres externes notés «a » (égalité) et «b» (coûts), est soumis aux contraintes topologiques de la catastrophe fronce. La fronce est une sorte de pli tridimensionnel. Elle se forme à la surface d'un feuillet qui représente les surfaces stables des attracteurs qui, en certains points (ensemble catastrophique), subissent les perturbations de la catastrophe (voir figure 1).

\section{Figure 1 : Représentation topologique du conflit des attracteurs dans le système de santé}

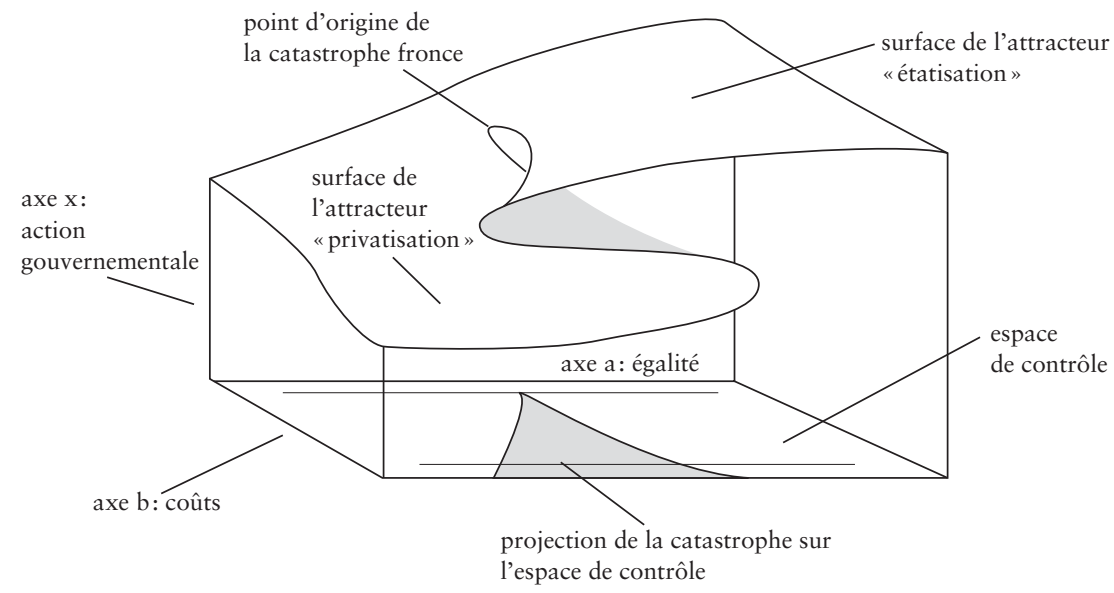

Il est à noter que pour Thom, tout conflit dans la nature qui oppose deux attracteurs dans un espace de contrôle à deux paramètres est défini topologiquement par la fronce ${ }^{13}$. En somme, la fronce est la dynamique globale — le «logos» pour parler autrement — qui contraint les actions locales

12. La liste et la définition mathématique de ces catastrophes élémentaires sont fournies par Thom dans l'ouvrage fondateur de sa théorie des modèles. Voir Thom, Stabilité structurelle et morphogenèse, p. 57-99.

13. Thom en fait la démonstration mathématique dans l'ouvrage précédemment cité, Stabilité structurelle et morphogenèse. À la page 62 de cet ouvrage, il expose d'ailleurs le 
du conflit, puisque «toute morphogenèse [est attribuée] à un conflit, à une lutte entre deux ou plusieurs attracteurs ${ }^{14}$. " Il faut donc considérer les catastrophes élémentaires de Thom comme des archétypes que les conflits de tendances réalisent, indépendamment du substrat matériel dans lequel ils s'incarnent. Ce qui change dans les différents substrats matériels, ce ne sont pas les dynamiques globales, mais plutôt les caractéristiques "internes" résultant notamment d'interactions stochastiques entre les composants élémentaires du substrat, comme par exemple la température du changement de phase d'un liquide.

Dans le contexte du conflit éthique examiné ici, le «substrat matériel » de l'action gouvernementale est constitué principalement par l'opinion publique et sa répartition obéissant aux lois normales (gaussiennes) de la statistique $^{15}$. Par ailleurs, puisque les catastrophes élémentaires sont associées à des potentiels, certaines règles supplémentaires s'appliquent au conflit pour en déterminer les manifestations visibles. Lorsque les deux attracteurs d'un système viennent en conflit près de l'ensemble catastrophique, il se crée sur la fonction qui les représente deux minima qui peuvent tous les deux constituer la partie visible du système. Pour mieux comprendre cette idée, prenons un exemple concret. Si on dépose une bille sur la surface illustrée dans la figure 1, on admet que cette bille, à un instant «t» donné, ne peut qu'occuper une seule position sur la surface. Considérons que cette position visible de la bille est l'action gouvernementale dans l'exemple du conflit éthique que j'ai choisi. Aux deux extrémités de la figure, un seul attracteur domine et la bille reste stable sur la surface de l'un de ces attracteurs. Mais si on déplace la bille vers la surface de l'ensemble catastrophique, il arrivera un moment où, à un instant donné, la bille pourra occuper deux positions dans la surface de la fronce: soit sur la partie du dessus appartenant un peu plus à la surface de l'attracteur «étatisation", soit sur la partie du dessous appartenant un peu plus à la surface de l'attracteur «privatisation». Étant donné que la bille ne peut qu'occuper une seule position à la fois, qu'est-ce qui déterminera le choix de la position? Lorsque les deux attracteurs luttent de cette manière, deux règles peuvent s'appliquer. La première est la règle de Maxwell. Selon cette règle, l'attracteur représentant le potentiel minimal prévaut. Autrement dit c'est, dans l'absolu, l'attracteur le plus fort qui devrait déterminer la manifestation visible du système ${ }^{16}$. Toutefois, cette règle ne tient pas compte de la situation locale du conflit, c'est-

théorème qui montre "qu'on peut identifier le type d'une catastrophe et son origine dynamique sans qu'on ait à connaître explicitement tous les paramètres internes dont dépend le système ».

14. Ibid., p. 327.

15. Voir Erik Christopher Zeeman, Catastrophe Theory (Selected Papers 1972-1977), London, Addison-Wesley Publishing Company, 1977, p. 304-306.

16. Dans notre exemple, l'attracteur le plus fort dépend de variables internes comme l'opinion publique. Ce sera donc l'attracteur le plus «populaire» qui sera le plus fort. Voir une application de ce principe dans Zeeman, Catastrophe Theory, p. 306-308. 
à-dire de sa situation actuelle déterminée par les événements passés. En pratique, il peut être difficile pour un gouvernement de passer directement d'un système de santé public à un système privé, et vice versa. Dans ces cas, c'est donc plutôt la règle du retard qui s'applique. Selon cette règle, lorsqu'un minimum de potentiel est atteint, c'est-à-dire lorsqu'un attracteur domine, cette situation est conservée tant qu'elle est stable, même si un autre minimum de potentiel existe ${ }^{17}$.

\subsection{Quelques hypothèses et énoncés résultant de l'application du modèle}

Comme je l'ai suggéré, les contraintes topologiques définies par le modèle favorisent la formulation d'hypothèses et d'énoncés relatifs au conflit éthique analysé. L'objectif de ces formulations est d'accroître l'intelligibilité afin d'aider à la prise de décision. Pour produire ces hypothèses et ces énoncés, on doit simplement tracer des chemins dans la figure topologique du conflit et les traduire en langage courant ${ }^{18}$. Le sens des formulations est le résultat d'un tracé mental effectué à partir de la figure 1 .

Voici quelques hypothèses et énoncés que suggère la dynamique globale du conflit éthique entre privatisation et étatisation:

- À coûts constants et bas, le désir d'un accès égalitaire aux soins poussera sans trop de problèmes l'action gouvernementale vers l'étatisation du système.

- Si le désir d'égalité est faiblement ancré dans la population, les coûts croissants du système pousseront presque invariablement l'action gouvernementale vers la privatisation.

- Dans une société où la notion d'égalité est relativement importante, l'augmentation croissante des coûts du système poussera le gouvernement dans une zone d'incertitude où son action peut basculer brutalement (ensemble catastrophique).

- Dans un contexte économique où les coûts du système sont élevés, il faut, si on veut préserver le système universel, favoriser dans la population l'éclosion d'un fort sentiment égalitaire.

- Si on veut faire avancer la cause de la privatisation du système de santé, alors il faut soit faire exploser les coûts, soit dévaloriser l'égalité au profit de la liberté individuelle.

- Si l'action gouvernementale se situe dans une zone de basculement catastrophique, il faut, pour ramener la stabilité, soit réduire les coûts du système, soit établir clairement la primauté de l'égalité ou de son opposé, la liberté individuelle.

- Etc.

17. Pour une présentation mathématique de ces règles, voir Ivar Ekeland, «La théorie des catastrophes ", La recherche, $\mathrm{n}^{\circ}$ 81, vol. 8, 1977, p. 745-754.

18. Voir d'autres exemples de ce procédé dans Zeeman, Catastrophe Theory, p. 334335. 
On remarque à la lecture des énoncés que leur validité est déterminée par la topologie du conflit. En ce sens, il est loisible d'en déduire que la topologie du processus participe à la genèse d'un espace sémantique "profond» qui donne un sens, qui éclaire le dilemme éthique. L'espace sémantique se définit comme un espace de qualités qui sert de support à la validité des énoncés produits par des locuteurs et à partir duquel sont extraits les concepts, puis les mots. C'est en quelque sorte un support référentiel. Il faut comprendre l'espace sémantique comme un feuillet cognitif - une carte de connexions neuronales - que le cerveau extrait à partir des données sensori-motrices liées à la perception par le sujet des formes du monde extérieur $^{19}$. La morphologie des phénomènes agit dans ce processus comme connecteur des feuillets entre eux. C'est en puisant dans cette morphologie que le cerveau détache un espace sémantique à partir des perceptions sensori-motrices, et c'est en puisant dans cet espace sémantique à base morphologique que le cerveau forme ensuite un concept qu'il désigne par un mot.

Autrement dit, tout se passe comme si se formait autour d'un noyau constitué par les morphologies du monde extérieur un ensemble de signes plus ou moins abstraits - les mots, les concepts - qui renvoient à la dynamique topologique d'une situation. La signification serait donc constituée d'un noyau topologique universel sur lequel viennent s'agréger des énoncés linguistiques culturellement variables. Dans l'un des ouvrages les plus importants consacrés à la pensée de René Thom, le mathématicien et philosophe français Jean Petitot affirme clairement le lien entre signification et topologie: "[L]a signification de ce mot n'est autre que la topologie globale du (ou des) attracteur(s) associé(s) et celle des catastrophes qu'ils subissent ${ }^{20}$.

La topologisation du sens qui opère dans la théorie thomienne des modèles explique la force de sa capacité descriptive. En sciences humaines et sociales, le langage n'agit bien souvent que sur un espace de description limité par le cloisonnement des principes et des concepts propres à une discipline, et dont l'utilisation est régulée par une logique classique d'identité et de non-contradiction. Et quand le langage quitte cet espace de description bien défini, comme dans la poésie par exemple, la description perd en clarté. Or la topologie des conflits à laquelle aboutit la procédure de modélisation thomienne ignore ces limites, ou plutôt elle les fait ressortir pour mieux les contourner:

En effet la pensée verbalisée a tendance à scléroser les concepts, liés qu'ils sont à un mot fixe du lexique: elle dissimule la variabilité intrinsèque derrière l'effet des auxiliaires et des fonctions de la grammaire. La T.C. permet une logique du continu, où l'on considère les concepts "variables" [...] Lorsque u décrit un chemin (uv) dans U, il est possible que le concept Fu se transforme continûment

19. Voir le détail de ce processus dans René Thom, Modèles mathématiques de la morphogenèse, Paris, Christian Bourgois, 1980, p. 298.

20. Jean Petitot, Morphogenèse du sens, Paris, P.U.F., 1985, p. 89. 
en un concept Gv dont la parenté avec Fu n'apparaîtra pas immédiatement, parce qu'en pensée normale il y a un seuil, un "tabou ", séparant u de v dans l'espace de contrôle U. La T.C. offre donc la possibilité (étendue) de transgresser le principe d'identité ${ }^{21}$.

Devant les luttes et les phénomènes discontinus qui surviennent dans un espace substrat donné et y apparaissent comme inexplicables ou irréconciliables, la théorie des modèles propose la création d'un espace de description multiplié où les éléments disjoints par le conflit se trouvent réunis. Cette idée peut être saisie à l'aide d'une analogie simple. Supposons deux feuilles de papier correspondant à deux plans distincts d'où je puis observer des phénomènes. Je dessine sur chacune des feuilles un point qui illustre le phénomène observé. Avec le langage ordinaire, il me sera difficile de trouver un espace sémantique conjoint dans lequel ces deux points seront mis en relation. Cette difficulté s'explique premièrement par les limites spatiales imposées par le système de référence de la langue de description. Ces limites imposent à la langue naturelle d'opérer à partir de concepts dérivés d'un espace sémantique spatialement défini. De plus, en lui-même, le concept est cognitivement plus étroit que l'espace sémantique associé à son objet. Il se rapporte exclusivement à sa définition dans un lexique, alors que l'espace sémantique peut inclure également des valeurs propulsées par les régions subcorticales du cerveau. Le concept fige la signification, ce qui rend l'analyse linguistique difficilement capable de saisir la dynamique et l'enchevêtrement des qualités qui sont à l'œuvre dans les phénomènes complexes.

À cause des limites du langage, l'explication causale de ces phénomènes discontinus aura recours, comme par réflexe, à des hypothèses de nature métaphysique - des concepts qui font appel à un «au-delà » de cet espace ici-bas - ou réductionniste — , une combinatoire d'entités élémentaires appartenant à un espace sémantique de niveau inférieur. Dans l'exemple que je donne, les deux points séparés peuvent être considérés dans un espace continu grâce à l'élaboration d'une géométrie dont les dimensions résultent de la multiplication de l'espace du plan des deux feuilles. Par la géométrisation du problème se crée alors un nouvel assemblage des espaces qui donne lieu à de nouvelles possibilités de description.

\subsection{L'exemple d'un modèle à trois ou quatre paramètres de contrôle}

D’aucuns pourraient se déclarer insatisfaits devant le modèle de la fronce, puisque celui-ci définit l'action gouvernementale seulement à l'aide de deux attracteurs qui renvoient à leur tour à deux paramètres de contrôle. Pourtant, pourrait-on objecter, l'action politique des gouvernements est souvent plus nuancée que celle proposée par le modèle de la fronce. Il s'y dessine habituellement des positions de compromis. Tout n'est pas toujours polarisé.

21. Thom, Modèles mathématiques de la morphogenèse, p. 124. 
Même s'il est clair que de nouveaux développements dans la théorie thomienne des modèles devront un jour survenir pour qu'elle soit généralisable à un ensemble de situations en sciences humaines et sociales, il reste que, dans son état actuel, elle offre un niveau de complexité déjà fort intéressant. Par exemple, il est possible à l'aide de la catastrophe papillon de définir une topologie à quatre paramètres de contrôle sur laquelle apparaît une zone de compromis dans la morphologie du conflit. La forme de la catastrophe papillon s'explique par la prise en considération de deux nouveaux paramètres de contrôle: "c », appelé par Zeeman facteur de biais (bias factor), et «d» qui est le temps ${ }^{22}$. Par l'addition de ces nouveaux paramètres, on ajoute à l'ensemble catastrophique une nouvelle complexité topologique qui laisse entrevoir un espace de l'action gouvernementale qui n'appartient plus exclusivement à l'un ou l'autre des attracteurs, mais à une zone de compromis qui se déploie entre les surfaces qui correspondent à l'étatisation et à la privatisation ${ }^{23}$.

L'ajout de nouveaux paramètres de contrôle accroît la stratification, au sens mathématique, de la morphologie de l'ensemble catastrophique. Conséquemment, l'espace occupé par cet ensemble peut être décomposé en un nombre supérieur de sous-espaces (des points, des arêtes, des surfaces, etc.) dont la codimension est généralement proportionnelle au degré d'instabilité de certains points critiques ${ }^{24}$. Les chemins tracés sur cette nouvelle topologie laissent envisager la possibilité d'une lecture plus fine et peut-être plus réaliste de l'action gouvernementale.

Dans l'approche de la modélisation catastrophiste adoptée par Zeeman ${ }^{25}$, le facteur de biais est un paramètre qui vient modifier les contraintes produites par la variation des paramètres "de base» que sont l'égalité et les coûts du système. Ce paramètre peut prendre la forme, par exemple, d'un facteur culturel ou d'un «effet de mode» qui infléchit le jugement des décideurs politiques et influe sur l'action gouvernementale. Pour notre exemple, je propose que ce facteur soit le sentiment de bien-être de la population. Cela veut dire, par exemple, que si les coûts du système sont incontrôlés et si la valeur d'égalité est constante, il se peut quand même, en présence d'un fort sentiment de bien-être au sein de la population, que la force d'inertie du gouvernement soit plus grande. Ce facteur vient donc donner un «biais» à l'action gouvernementale. Quant au dernier paramètre, appelé par Zeeman

22. Zeeman, Catastrophe Theory, p. 344-345.

23. La forme de la catastrophe papillon est beaucoup plus complexe que celle de la fronce. Pour des raisons techniques, je n'en fournis pas une illustration dans cet article. Pour d'autres détails, voir entre autres Zeeman, Catastrophe Theory, p. 338.

24. En dimension finie, la codimension est égale à la différence de la dimension d'un espace par la dimension de l'un de ses sous-espaces. Pour l'explication mathématique, voir par exemple Jean Petitot, "Approche morphodynamique de la formule canonique du mythe", L'Homme, 106-107, XXVIII (2-3), p. 34-36.

25. Zeeman, Catastrophe Theory, p. 338-341. 
facteur papillon - à cause de la forme caractéristique qu'il donne à la catastrophe -, il correspond au temps et à son influence sur le processus de décision. Généralement, et ce fait est largement reconnu dans la culture populaire ${ }^{26}$, le temps a pour effet d'adoucir les positions extrêmes et de les ramener vers la zone de compromis qui se creuse au cœur de la catastrophe papillon.

\section{Quelques avancées philosophiques permises par la théorie thomienne des modèles}

Ainsi que je l'ai évoqué précédemment, il existe deux modes d'application de la théorie des modèles chez Thom. Dans la première, "on part de principes et de lois physiques pour en dériver les équations du système ou du processus considéré, et l'on cherche à déduire les infrastructures catastrophiques à partir de dynamiques internes explicites. Bref, l'on dérive (causalement) l'apparaître morphologique de l'être physique ${ }^{27}$ ». La deuxième voie, qui procède en sens inverse, est celle de Platon dans l'allégorie de la caverne. Bien qu'extrêmement intéressante pour la philosophie, cette voie a subi les critiques les plus virulentes, notamment celles de Sussman et Zahler dans un article substantiel ${ }^{28}$.

Au-delà des critiques plus techniquement mathématiques que nous ne souhaitons pas aborder dans le cadre de cet article, il est apparu clair pour ses pourfendeurs que la théorie thomienne des modèles posait problème, surtout dans les applications dues à Zeeman ${ }^{29}$. Par exemple, on peut juger un peu arbitraire l'imposition, sur la toile complexe des conflits éthiques, d'une morphologie mathématique transcendante censée en expliquer les "contraintes». Tout comme on peut estimer qu'il est impossible de produire des algorithmes de décision satisfaisants à partir d'un nombre très limité d'infrastructures catastrophiques élémentaires ${ }^{30}$. Ce scepticisme est sans doute aussi renforcé par la technique de modélisation qui choisit, par tâtonnement, les paramètres sur lesquels on projette le conflit d'attracteurs.

26. «Le temps arrange les choses ", entend-on souvent à ce sujet.

27. Petitot, Logos et théorie des catastrophes, p. 46.

28. Sussman, Hector et Zahler, Raphael, "Catastrophe theory as applied to the social and biological sciences: a critique ", Synthese, vol. 37, n 2, 1978, p. 117-216.

29. Je ne m'étendrai pas sur la critique la plus récurrente de la théorie, celle où on lui reproche son incapacité prédictive. La réponse de Thom à cette critique est abondamment documentée. Elle explique d'ailleurs le titre de l'ouvrage Prédire n'est pas expliquer, précédemment cité. Voir entre autres Thom, Prédire..., p. 43-44.

30. À l'échelle macroscopique, les limites perceptuelles des sens ne permettent pas une observation fine des phénomènes, c'est pourquoi il est possible de réduire le nombre de catastrophes observées à des conflits d'un maximum de trois ou quatre attracteurs. Cette simplification correspond en fait au "découpage" naturel qu'effectue le regard de l'observateur dans la complexité mouvante des phénomènes. 
Certaines de ces critiques sont sans doute légitimes, et je suis le premier à reconnaître qu'en certaines de ses applications la théorie thomienne des modèles doit encore faire l'objet d'un effort supplémentaire de systématisation. Mais en rester à ce scepticisme satisfait, c'est ignorer les remarquables avancées philosophiques qu'elle laisse espérer ${ }^{31}$. D'abord, la théorie des modèles de Thom s'appuie sur un postulat fort. Elle stipule que les conflits d'attracteurs et les phénomènes critiques correspondent nécessairement à des morphologies typiques ou archétypiques — les catastrophes - qui président à l'organisation des substrats matériels, y compris le cerveau ${ }^{32}$. De cette façon, la pensée de Thom comble le fossé épistémologique qui se creuse entre le sujet et l'objet. En affirmant la primauté et l'universalité des êtres géométriques dans l'organisation de la matière et de la pensée, l'épistémologie thomienne laisse entendre que la reconstruction mentale d'ensembles catastrophiques qui englobent les discontinuités locales des phénomènes renvoie à la structuration objective du réel. Autrement dit, l'effort de modélisation par la pensée est en fait une redécouverte de la topologie universelle qui gouverne le monde visible et lui assigne des finalités que le réductionnisme masque sous le voile des causes matérielles. En termes clairs, la topologie des catastrophes établit des invariants de la perception qui conditionnent à la fois l'apparition objective des phénomènes ainsi que leur intégration dans l'espace sémantique d'un sujet ${ }^{33}$.

En outre, le principe de justification que favorise l'épistémologie thomienne ne me paraît pas appartenir aux versions traditionnelles du fondationnalisme et du cohérentisme, car il récuse le langage dans sa primauté sémantique. Dans la pensée de Thom, les frontières des concepts, sur lesquels se base habituellement la justification, se trouvent modifiées par leur renvoi à la dynamique des variations que décrit la modélisation catastrophiste. Même si Thom lui-même ne s'est jamais beaucoup avancé sur le terrain de l'éthique, sa pensée semble vouloir obéir à une forme de réalisme moral que ne renieraient sans doute pas les philosophes grecs. Puisque le monde existe et «se tient " grâce à des propriétés topologiques déterminées, il inscrit le devenir des êtres qui le composent dans des processus structurels fortement finalisés - des prégnances - auxquels peuvent être rattachées des actions et des valeurs dont la justification repose sur l'adéquation à la

31. Voir Krzysztof Pomian, "La philosophie de René Thom», Logos et théorie des catastrophes, Genève, Éditions Patino (coll. «Colloque de Cerisy»), 1988, p. 319-334.

32. À ce sujet, Thom affirme clairement son opposition au matérialisme d'un Jean-Pierre Changeux. À l'argument des matérialistes qui déclarent que si je détruis la matière du cerveau, il n'y aura plus de pensée, Thom rétorque qu'on peut très bien imaginer qu'en détruisant le cerveau, c'est en fait la structure formelle de son organisation que je détruis, et que c'est au fond cela qui m'empêche ou me permet de penser. Voir Thom, Prédire n'est pas expliquer, p. 87-88.

33. Petitot, Morphogenèse du sens, p. 87-88. 
«cartographie» naturelle de ce monde. Dans une recherche ultérieure, il serait sans doute intéressant de pousser plus loin cette question.

Par ailleurs, il importe d'insister sur un point: le présupposé épistémologique d'une organisation géométrique du réel est ontologiquement neutre ${ }^{34}$. Pour Thom, le problème de savoir si des entités immatérielles existent " avant » ou «au-delà» de la matière est secondaire ${ }^{35}$. Ce qui compte, c'est de comprendre que la capacité que possède le regard humain d'identifier, de classer et de décrire linguistiquement les phénomènes dépend d'une stabilité structurelle dont certaines conditions et limites sont énoncées par la théorie thomienne des modèles. En ce sens, cette théorie est une théorie générale de la connaissance «naïve ${ }^{36}$. Pour ces raisons, plusieurs commentateurs, dont Jean Petitot, n'ont cessé de rappeler que l'un des apports philosophiques majeurs de la pensée de Thom est de réconcilier le regard phénoménologique posé sur la surface des choses et le regard scientifique fondé sur la découverte de leur «intériorité » mathématique ${ }^{37}$.

De plus, la théorie des modèles généralise l'usage d'une approche épistémologique fondée sur l'analogie. Elle établit que les règles d'association qui permettent à la pensée de relier des termes indépendants sont fondées sur des isomorphismes ${ }^{38}$. Je peux par exemple associer le terme «révolution» à une «rupture brutale de l'ordre établi » seulement si je possède implicitement l'idée d'une forme dont les bords se transforment pour en modifier les caractéristiques apparentes. Alors, face à l'inconnu et à l'indéterminé, l'analogie se sert des formes archétypiques de la topologie pour regrouper les données empiriques apparaissant sur un espace sémantique avec celles qui apparaissent sur un autre. De cette manière, la connaissance saisit l'inconnu à partir du connu, et part du local pour aller vers le global.

Comme je l'ai indiqué plus haut, ces formes typiques sont en nombre restreint, ce qui limite apparemment la finesse de leur pouvoir d'intelligibilité sur les conflits éthiques. Si l'on s'en tient aux applications proposées par Zeeman, je serais porté à croire qu'effectivement leur pouvoir est limité, du moins en l'absence de nouveaux développements mathématiques catastrophistes. Pourtant, il ne faut pas perdre de vue que tout un programme de recherche peut s'ouvrir si on considère que les catastrophes élémentaires servent de fondement à une grammaire actantielle ${ }^{39}$ qui reproduit les conflits surgissant sur l'espace-temps à l'aide notamment des morphologies-

34. Ibid., p. 79-80.

35. Thom, Prédire n'est pas expliquer, p. 88-89.

36. J'entends par connaissance naïve la connaissance du monde familier à l'aide de l'observation et du langage ordinaires.

37. Voir Jean Petitot, "Note sur la querelle du déterminisme», dans Krzysztof Pomian (dir.), La querelle du déterminisme, Paris, Gallimard (coll. «Le débat»), 1990, p. 220-221.

38. Vient du mot «isomorphe» qui signifie «de même forme».

39. Les actants dans la linguistique thomienne correspondent à des formes stables qui se distinguent dans l'espace de la description. Dans la phrase «Le chat surveille la souris », le chat 
archétypes de la langue naturelle ${ }^{40}$. Au nombre de seize, ces morphologies pourraient probablement être combinées et intégrées à une sorte de sémantique topologique applicable à la théorie éthique. Aux contraintes topologiques très générales opérant sur le réel à partir des catastrophes élémentaires pourrait s'ajouter un niveau intermédiaire de modélisation intervenant cette fois sur le langage et non plus simplement sur la morphologie des phénomènes. Les morphologies d'événements viendraient ainsi façonner la syntaxe structurale universelle des langues naturelles par des contraintes qui s'imprègnent dans la langue des acteurs d'un conflit et clôturent leurs pensées et leurs actions dans un horizon des possibles ${ }^{41}$. À mon sens, l'intérêt d'une telle entreprise serait d'universaliser les principes de justification des décisions éthiques, et de contourner ainsi le biais culturel et le flou sémantique qui sous-tendent les approches canoniques, en bioéthique et en éthique de la santé publique notamment.

\section{Conclusion}

Deux objectifs ont guidé ma réflexion tout au long de cet article. Celui, d'abord, de trouver pour la philosophie de nouveaux outils méthodologiques et théoriques lui ouvrant la possibilité de contribuer par des moyens extralinguistiques à la prise de décision en éthique; celui, ensuite, d'esquisser les contours d'un programme de recherche multidisciplinaire par lequel la philosophie pourra, à terme, participer de façon significative à la gestion de la complexité dans différents domaines. Car j'ai acquis la conviction qu'il deviendra de plus en plus difficile, pour la philosophie et les sciences humaines en général, de gérer le flot d'informations dans lequel nous plonge la culture contemporaine mondialisée à l'aide des concepts issus de la langue usuelle. Si elle ne veut pas se marginaliser encore davantage, la philosophie devra, me semble-t-il, se transformer, et surtout faire évoluer les formes de son langage et de sa pensée. Même si au regard de ces objectifs la théorie thomienne des modèles ne constitue qu'une base de travail, elle n'en fournit pas moins l'exemple éloquent d'une transformation de la pensée philosophique par l'intégration d'idées mathématiques. D'ailleurs, les connaissances actuelles nous permettront sans doute de pousser ce programme un peu plus loin.

En somme, la pensée de Thom indique la voie d'un rapprochement possible entre des niveaux de conceptualisation qui s'étendent de l'expérience immédiate de notre environnement à la construction de modélisations

et la souris sont des actants. Dans la grammaire actantielle, les dynamiques globales sont associées à des verbes fondateurs, alors que les actants sont décrits par des substantifs.

40. Voir Thom, Stabilité structurelle et morphogenèse, p. 310-315.

41. L'un des aspects originaux de cette approche est de lier la formalisation par la syntaxe structurale à des représentations sémantiques qui, en raison de leur ancrage topologique, conservent leur universalité. 
de plus en plus abstraites qui se traduiront en fin de compte par des actions nouvelles sur le monde qui nous entoure. En ce sens, les positions philosophiques qui se profilent en arrière-plan de la théorie thomienne des modèles me paraissent être fidèles à l'esprit même de la philosophie telle que la concevait Platon dans son allégorie de la caverne. Elles font œuvre de libération pour ceux parmi nous que les chaînes de la connaissance et de limitation perceptuelle attachent à la surface des objets familiers décrits par le langage ordinaire. Pour ces prisonniers qui ne disposent pas de moyens techniques impressionnants, si ce n'est la force de leur regard, la philosophie de Thom offre une voie épistémologique de salut. En récusant l'omnipotence méthodologique du réductionnisme, qui oblige la connaissance à dévoiler le secret sans fond des entités élémentaires, René Thom nous rappelle, à l'instar de Platon jadis, que pour peu qu'il consente à devenir géomètre, le penseur du quotidien et du familier peut lui aussi mieux comprendre pour mieux agir. 